\title{
Development of microsatellite multiplexes for wild goats using primers designed from domestic Bovidae
}

\author{
Célia Maudet*, Gordon Luikart, Pierre TABerlet \\ Laboratoire de biologie des populations d'altitude (Cnrs - UMR 5553), \\ Université Joseph Fourier, BP. 53, 38041 Grenoble Cedex 9, France
}

\begin{abstract}
Many wild goat taxa (Capra spp.) are endangered and would benefit from the availability of molecular tools that are useful for population management and conservation. We developed microsatellite DNA markers useful in all wild goat species, by using a cross-species amplification approach. Seventy-five microsatellite primer pairs designed from domestic cattle (Bos taurus), sheep (Ovis aries) and goat (Capra hircus) were tested on three distantly related Capra species: C. ibex ibex, C. [i.] sibirica, and C. pyrenaica. On average, $90 \%$ of the domestic ungulate primers amplified a microsatellite PCR product in the wild goat species. Forty percent of the total were polymorphic in $C$. $i$. ibex, which is expected to have the lowest genetic diversity among all Capra species. We developed multiplexes of 24 polymorphic fluorescent microsatellite loci that can be amplified in 13 PCR reactions and loaded into two gel-lanes. These microsatellites will allow studies of conservation and population ecology in all Capra species, and the multiplexes will reduce the time and cost of the genetic analyses.
\end{abstract}

Capra / cross-species amplification / ungulate conservation / fluorescent multiplexes

Résumé - Développement de multiplexes microsatellites pour les Capra sauvages à partir d'amorces définies chez les Bovidea domestiques. Plusieurs espèces sauvages du genre Capra sont considérées comme menacées et requièrent un outil moléculaire fiable pour les études de gestion des populations et de conservation. Un ensemble de marqueurs microsatellites utilisable pour l'ensemble des espèces du genre Capra a été développé en employant l'approche de transfert inter-spécifique d'amorces microsatellite. Soixante-quinze couples d'amorces microsatellite définies sur les bovins (Bos taurus), ovins (Ovis aries) et caprins (Capra hircus) domestiques ont été testés sur trois espèces génétiquement distantes du genre Capra: C. ibex ibex, C. [i.] sibirica, and C. pyrenaica. Quatre-vingt dix pour-cent de ces amorces ont conduit à l'obtention d'un produit d'amplification spécifique. Quarante pour cent de ces

* Correspondence and reprints

E-mail: cmaudet@ujf-grenoble.fr 
marqueurs se sont révélés polymorphes chez Capra. $i$. ibex, espèce estimée la moins variable de l'ensemble du genre Capra. Un système de multiplexes a été développé afin d'amplifier 24 loci polymorphes au cours de 13 réactions PCR indépendantes, et de les charger dans deux pistes de séquenceur automatique. Ces microsatellites donnent la possibilité d'études de conservation et d'écologie sur l'ensemble des espèces du genre Capra, et les multiplexes développés permettent une réduction du coût et du temps de ces analyses génétiques.

Capra / transfert inter-spécifique d'amorces / conservation des ongulés / multiplexes fluorescents

\section{INTRODUCTION}

At the present time, 5-8 wild goat species (Capra spp., taxonomy varies among authors) have been described and several of them are endangered [22]. Their geographic distribution includes mountainous regions in Europe, Africa and Asia. Many populations are becoming fragmented and reduced in size, leading to an increased risk of extinction [1]. Studies in conservation and population biology will require molecular markers with a high degree of variability and that can be used in all Capra species (i) to measure and monitor levels of genetic variation, (ii) to assess gene flow and population structure; and (iii) to conduct forensic investigations. During the last decade, microsatellite markers have proven to be a useful tool for conservation and population ecology studies in diverse wild animal species $[4,11,18]$. Microsatellites are excellent genetic makers because of their high polymorphism, abundance and broad distribution in mammalian genomes. Generally, microsatellites are isolated by constructing and screening a genomic DNA library, DNA sequencing and designing of primers (e.g. [29]). However, this method is relatively laborious and expensive. In addition, finding polymorphic microsatellites in species with reduced polymorphism can be especially difficult.

Another approach consists in exploiting microsatellites developed from closely related species. Many studies $[7,16,17,20,21,29,30]$ have demonstrated that microsatellite flanking sequences are greatly conserved across artiodactyl species. Thus primers defined for one species often can be used to amplify microsatellites in other taxa. The great number of PCR primers designed for domestic cows, sheep or goats could be employed to amplify microsatellites in wild goat species. This approach presents many advantages. The most notable is that many microsatellites have already been isolated and are easily available in publications and on web sites (currently, several thousand bovine, ovine and caprine microsatellites are known and numerous web sites provide easy access to primer sequences). This accessibility allows the testing of many markers and the ability to choose markers with desirable characteristics: (i) the size of the amplified DNA fragments (in order to load several loci into the same gel well: multiplex loading), (ii) the chromosome location (to select loci across different 
chromosomes and avoid linked loci, knowing marker locations are very similar across ungulates [28]), (iii) the ability to amplify in all interested species, and (iv) the polymorphism level appropriate for the study of interest. This approach will often be better suited to inter-specific studies than the cloning approach. It is less expensive, faster and allows the choice of loci depending on the requirements of the study.

The aims of the present study were to find microsatellite loci developed in the abundant studies of domestic Bovidae (cattle, sheep and goat) that could amplify polymorphic microsatellites across wild goat species. Seventyfive microsatellites from domestic ungulates were tested on three species of Capra, including the most genetically divergent species, Siberian ibex (Capra [ibex] sibirica), the severely bottlenecked Alpine ibex (Capra ibex ibex) and the endangered Spanish ibex (Capra pyrenaica) [10]. We developed multiplexes of 24 microsatellite loci that can be amplified in 13 PCR reactions and loaded into two gel-lanes.

\section{METHODS}

\subsection{Samples}

Tissue or blood samples were collected from captured or hunted individuals. We analyzed four samples of Capra [i.] sibirica from distant locations in Mongolia, four samples of Capra pyrenaica from distant populations in Spain (including two subspecies; C. p. hispanica and C. p. victoriana), and 36 Capra ibex ibex from the Gran Paradiso population (Italy). DNA was extracted using the QIAamp Tissue or Blood Kit (Qiagen) following the manufacturer's procedures.

\subsection{Loci tested}

In order to identify reliable loci, we tested microsatellites known to work well and be polymorphic in domestic ungulates. We tested seventy-five microsatellite primer pairs designed in domestic cattle, goat or sheep: $\mathrm{BM} 1818^{\mathrm{p}}$,

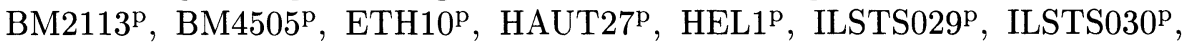
INRABERN175 ${ }^{\mathrm{p}}$, INRA011 $^{\mathrm{p}}$, INRA040 $^{\mathrm{p}}$, INRABERN185 ${ }^{\mathrm{p}}$ MILSTS076 $^{\mathrm{p}}$, OarFCB193 ${ }^{\mathrm{p}}, \quad$ OarAE54 $^{\mathrm{p}}, \quad$ OarFCB $48^{\mathrm{p}}, \quad$ SR-CSRP-15 ${ }^{\mathrm{p}}$, SR-CSRP-23 $^{\mathrm{p}}$, SR-CSRP-24 ${ }^{\mathrm{p}}$, SR-CSRP-25 ${ }^{\mathrm{p}}$, SR-CSRP-26 $^{\mathrm{p}}$, SR-CSRP-6 $^{\mathrm{p}}$, SR-CSRP-7 $^{\mathrm{p}}$, SR-CSRP-8 ${ }^{\mathrm{p}}$, SR-CSRP-9 $^{\mathrm{p}}$, TGLA122 $^{\mathrm{p}}$, TGLA126 $^{\mathrm{p}}$ TGLA227 $^{\mathrm{p}}$, URB058 $^{\mathrm{p}}$, $\mathrm{BM}_{143^{\mathrm{m}}}, \mathrm{BM} 1443^{\mathrm{m}}, \mathrm{BM} 1500^{\mathrm{m}}, \mathrm{BM} 1824^{\mathrm{m}}, \mathrm{BM} 203^{\mathrm{m}}, \mathrm{BM}^{2078^{\mathrm{m}}}, \mathrm{BM}{ }^{2} 37^{\mathrm{m}}$,

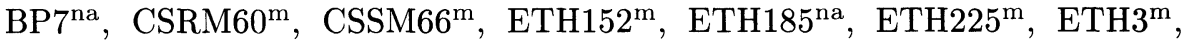

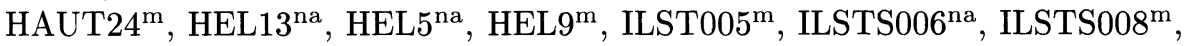
ILSTS011 $^{\mathrm{m}}, \operatorname{ILSTS}_{097^{\mathrm{m}}}$, INRA005 $^{\mathrm{m}}$, INRA023 $^{\mathrm{m}}$, INRA032 $^{\text {na }}, \operatorname{INRA035}^{\text {na }}$, INRA037 $^{\mathrm{m}}$, INRA063 $^{\mathrm{m}}$, INRABERN172 $^{\mathrm{m}}, \mathrm{MAF} 209^{\mathrm{m}}, \mathrm{MAF}^{\mathrm{m}}{ }^{\mathrm{m}}$, MCHII-DR $^{\mathrm{m}}$, 
MM12 ${ }^{\mathrm{m}}$, OarFCB20 ${ }^{\mathrm{m}}$, SPS $15^{\text {na }}$, SR-CSRP- ${ }^{\mathrm{m}}$, SR-CSRP- $11^{\mathrm{m}}$, SR-CSRP$12^{\mathrm{m}}$, SR-CSRP- $13^{\mathrm{m}}$, SR-CSRP-3 ${ }^{\mathrm{m}}$, SR-CSRP- $4^{\mathrm{m}}$, SR-CSRP- $^{\mathrm{m}}$, TGLA $^{2} 3^{\mathrm{m}}$, $\mathrm{URB} 56^{\mathrm{m}}$ (p: polymorphic loci, m: monomorphic loci, na: non-amplified loci).

\subsection{PCR conditions}

Amplifications were performed in a total reaction volume of $25 \mu \mathrm{L}$ containing $3 \mu \mathrm{L}$ of DNA (1-10 ng), 0.1 to $1 \mu \mathrm{M}$ of each primer (one to three loci were amplified in the same reaction), $100 \mu \mathrm{M}$ dNTP, $2.5 \mathrm{mM} \mathrm{MgCl}_{2}$, PCR buffer $(10 \mathrm{mM}$ Tris- $\mathrm{HCl}(\mathrm{pH} 8.3), 50 \mathrm{mM} \mathrm{KCl}), 5 \mathrm{ng}$ of BSA, $1 \mathrm{U}$ of AmpliTaq Gold $^{\mathrm{TM}}$-Polymerase (Perkin Elmer). PCR amplifications were conducted in a Perkin Elmer 9700 thermal cycler using the following conditions: an initial denaturation step at $95{ }^{\circ} \mathrm{C}$ for 10 min followed by 10 cycles of $30 \mathrm{~s}$ at $95{ }^{\circ} \mathrm{C}$, $30 \mathrm{~s}$ at $65^{\circ} \mathrm{C}, 60^{\circ} \mathrm{C}$ or $55^{\circ} \mathrm{C}$ with one degree reduction at each cycle and $60 \mathrm{~s}$ at $72{ }^{\circ} \mathrm{C}$, followed by 30 cycles of $30 \mathrm{~s}$ at $95{ }^{\circ} \mathrm{C}, 30 \mathrm{~s}$ at $55^{\circ} \mathrm{C}, 50{ }^{\circ} \mathrm{C}$ or $45^{\circ} \mathrm{C}$ and $60 \mathrm{~s}$ at $72{ }^{\circ} \mathrm{C}$. For each of the 75 microsatellite primer pairs, the three annealing temperatures were tested to obtain PCR products (Tab. I).

\subsection{Multiplex PCR and loading}

In order to reduce the cost and the time of the genotypic procedure, we identified groups of 2-3 loci that co-amplify in the same PCR reaction (at the same temperature) without inhibition or artifacts. We amplified only 23 loci per PCR to ensure error-free genotypes when using a range of sample types and quality (hairs, feces, skins, blood...). We tested different primer concentrations to determine the optimum for amplification. PCR products were electrophoresed using an ABI Prism 377 automated sequencer (Perkin Elmer) that detects three different color-labeled primers. Considering the size and the labeled color of PCR product, it is possible to load several loci in the same sequencer well.

\section{RESULTS AND DISCUSSION}

Of the 75 microsatellite primer pairs tested, 67 amplified a specific PCR product and produced a typical microsatellite pattern for all three Capra species. Eight primer pairs failed to amplify Capra DNA (BP7, ETH185, HEL13, HEL5, ILSTS006, INRA032, INRA035, SPS115). Among the 67 loci, 29 were polymorphic in Capra i. ibex (expected to be the least variable Capra species because 
of its history of severe bottlenecks [24]). Indeed, the average heterozygosity and number of alleles per locus was low (0.44 and 2.7, respectively, for the polymorphic loci) and comparable to that in other severely bottlenecked mammal species $[8,26]$. Given this reduced polymorphism, the cloning approach for developing microsatellites would have been relatively difficult and expensive. Another study of the same Gran Paradiso population found even lower heterozygosity (mean $\mathrm{H}=0.19$ ) [21]. However, we screened more loci, used some different microsatellites and sampled from more distant locations within the Gran Paradiso National Park.

The 24 most polymorphic loci were selected to establish multiplexes for C. $i$. ibex. The microsatellite loci were amplified in 13 independent PCR reactions and were electrophoresed in two lanes (i.e., two groups of 12 loci were loaded in two sequencer wells) (Tab. I). A potential problem with the crossamplification approach is that primer sequences occasionally are not conserved and some alleles are not amplified. However, all loci were in Hardy-Weinberg proportions (tested in the 36 C. $i$. ibex individuals from the Grand Paradiso population) and there was no tendency toward a deficit (or excess) of heterozygotes. Although some loci are on the same chromosome (Tab. II), no significant linkage disequilibrium was detected (using the 36 C. $i$. ibex individuals).

The multiplexes were developed for $C$. $i$. ibex but could be used for other Capra species with few modifications (see allele lengths in Tab. II). All 24 polymorphic loci in C. i. ibex were also polymorphic in at least one of the two other species (Tab. II). In fact, all loci were polymorphic in C. i. sibirica (and some even had 5-7 alleles) even though we genotyped only four individuals. Obviously, if we had typed many more individuals we would have found even more alleles.

These polymorphic microsatellites amplified reliably in two of the most genetically divergent Capra species, so, all wild goat species could probably be studied with these markers. The availability of polymorphic molecular makers is essential for wild goat management because several are endangered and most have a highly fragmented population structure [22]. Furthermore, nearly all Capra species suffer from poaching, which could be combated using microsatellites and forensic investigations [25]. The loci identified here will be useful for studies of behavioral and population ecology as well as for the management and conservation of all Capra species.

\section{ACKNOWLEDGEMENTS}

We thank B. Bassano (Parco Nazionale Gran Paradiso, Italy) and Y. Zagdsuren for providing samples. Funding was provided by the BRG (Bureau des ressources génétiques) and INTERREGII (Vanoise/Gran Paradisio). 
Table I. Groups of loci for multiplex PCR and multiplex loading in Capra i. ibex. The chromosome numbers are for goats (but are usually the same for cattle: Vaiman et al. 1996). Exponent letters indicate multiplex PCR, exponent numbers indicate loci multiplex loaded together in one well.

\begin{tabular}{|c|c|c|c|c|c|c|}
\hline Locus & Reference & Primers sequences $\left(5^{\prime}-3^{\prime}\right)$ & Origin & $\begin{array}{l}\text { Chrom. } \\
\text { (in goats) }\end{array}$ & $\begin{array}{l}\text { Primer } \\
\text { Conc. } \\
(\mu \mathrm{M})\end{array}$ & $\begin{array}{l}\text { Annealing } \\
\text { temp. }\left({ }^{\circ} \mathrm{C}\right)\end{array}$ \\
\hline OarFCB0193 ${ }^{1 a}$ & {$[5]$} & $\begin{array}{l}\text { GTTTCTTTTCATCTCAGACTGGGATTCAGAAAGGC } \\
\text { GCTTGGAAATAACCCTCCTGCATCCC }\end{array}$ & Ovine & 11 & 0.2 & 55 \\
\hline OarFCB $48^{1 a}$ & {$[6]$} & $\begin{array}{l}\text { GACTCTAGAGGATCGCAAAGAACCAG } \\
\text { GAGTTAGTACAAGGATGACAAGAGGCAC }\end{array}$ & Ovine & 17 & 0.4 & 55 \\
\hline SR-CRSP-26 ${ }^{1 b}$ & {$[31]$} & $\begin{array}{l}\text { ACAGAGGTGAAGAATAAGGAGAGTG } \\
\text { GATAGTTTCAGAAGACCCAGTTGAG }\end{array}$ & Caprine & Unknown & 0.1 & 55 \\
\hline ETH10 $1 \mathrm{~b}$ & {$[23]$} & $\begin{array}{l}\text { GTTCAGGACTGGCCCTGCTAACA } \\
\text { ССТCCAGCССАСТTTCTСТTСТC }\end{array}$ & Bovine & 5 & 0.3 & 55 \\
\hline ILSTS029 $^{1 \mathrm{c}}$ & {$[14]$} & $\begin{array}{l}\text { TGITTTGATGGAACACAGCC } \\
\text { TGGATTTAGACCAGGGTTGG }\end{array}$ & Ovine & 3 & 0.2 & 55 \\
\hline $\mathrm{BM}_{1818^{1 \mathrm{~d}}}$ & {$[3]$} & $\begin{array}{l}\text { AGCTGGGAATATAACCAAAGG } \\
\text { AGTGCTTTCAAGGTCCATGC }\end{array}$ & Bovine & 23 & 0.4 & 50 \\
\hline INRABERN185 $1 \mathrm{~d}$ & {$[12]$} & 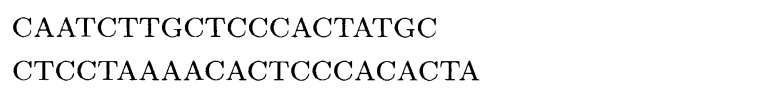 & Bovine & 18 & 0.2 & 50 \\
\hline $\mathrm{BM} 2113^{1 \mathrm{e}}$ & [3] & $\begin{array}{l}\text { GCTGCCTTCTACCAAATACCC } \\
\text { CTTCCTGAGAGAAGCAACACC }\end{array}$ & Bovine & 2 & 0.2 & 50 \\
\hline
\end{tabular}


Table I. Continued.

\begin{tabular}{|c|c|c|c|c|c|c|}
\hline Locus & Reference & Primers sequences $\left(5^{\prime}-3^{\prime}\right)$ & Origin & $\begin{array}{l}\text { Chrom. } \\
\text { (in goats) }\end{array}$ & $\begin{array}{l}\text { Primer } \\
\text { Conc. } \\
(\mu \mathrm{M})\end{array}$ & $\begin{array}{l}\text { Annealing } \\
\text { temp. }\left({ }^{\circ} \mathrm{C}\right)\end{array}$ \\
\hline SR-CRSP-25 ${ }^{1 \mathrm{f}}$ & {$[31]$} & $\begin{array}{l}\text { AACTATAACGGGAAGGAGTCTGG } \\
\text { AGGTTGTAGGAGTCGGACACAG }\end{array}$ & Caprine & Unknown & 0.2 & 45 \\
\hline HEL $1^{1 f}$ & {$[13]$} & $\begin{array}{l}\text { CAACAGCTATTTAACAAGGA } \\
\text { AGGCTACAGTCCATGGGATT }\end{array}$ & Bovine & 15 & 0.5 & 45 \\
\hline SR-CRSP-6 ${ }^{1 \mathrm{~g}}$ & {$[2]$} & $\begin{array}{l}\text { CATAGTTCATTCACAATATGGCA } \\
\text { GTTTCTTCATGGAGTCACAAAGAGTTGAA }\end{array}$ & Caprine & Unknown & 0.2 & 45 \\
\hline SR-CRSP-9 $9^{1 \mathrm{~g}}$ & [2] & $\begin{array}{l}\text { AGAGGATCTGGAAATGGAATC } \\
\text { TGGTGGTTCTGTGAAGATTTG }\end{array}$ & Caprine & 12 & 0.5 & 45 \\
\hline OarAE054 ${ }^{2 h}$ & [19] & $\begin{array}{l}\text { TACTAAAGAAACATGAAGCTCCCAC } \\
\text { GGAAACATTTATTCTTATTCCTCAGTG }\end{array}$ & Ovine & Unknown & 0.1 & 55 \\
\hline TGLA122 $2 \mathrm{~h}$ & [9] & $\begin{array}{l}\text { CCCTCCTCCAGGTAAATCAGC } \\
\text { AATCACATGGCAAATAAGTACATAC }\end{array}$ & Bovine & 21 & 0.2 & 55 \\
\hline HAUT272i & {$[27]$} & $\begin{array}{l}\text { TTTTATGTTCATTTTTTGACTGG } \\
\text { AACTGCTGAAATCTCCATCTTA }\end{array}$ & Bovine & 26 & 0.5 & 50 \\
\hline ILSTS $030^{2 j}$ & {$[14]$} & $\begin{array}{l}\text { CTGCAGTTCTGCATATGTGG } \\
\text { GTTTCTTCTTAGACAACAGGGGTTTGG }\end{array}$ & Ovine & 2 & 0.1 & 55 \\
\hline
\end{tabular}


Table I. Continued.

\begin{tabular}{lllllll}
\hline Locus & Reference & Primers sequences $\left(5^{\prime}-3^{\prime}\right)$ & Origin & $\begin{array}{l}\text { Chrom. } \\
\text { in goats })\end{array}$ & $\begin{array}{l}\text { Primer } \\
\text { Conc. } \\
(\mu \mathrm{M})\end{array}$ & $\begin{array}{l}\text { Annealing } \\
\text { temp. }\left({ }^{\circ} \mathrm{C}\right)\end{array}$ \\
\hline SR-CRSP-8 $^{\mathbf{2 j}}$ & {$[2]$} & $\begin{array}{l}\text { TGCGGTCTGGTTCTGATTTCAC } \\
\text { CCTGCATGAGAAAGTCGATGCTTAG }\end{array}$ & Caprine & Unknown & 0.5 & 55 \\
\hline SR-CRSP-24 $^{\mathbf{2 k}}$ & {$[31]$} & $\begin{array}{l}\text { AGCAAGAAGTGTCCACTGACAG } \\
\text { TCTAGGTCCATCTGTGTTATTGC }\end{array}$ & Caprine & Unknown & 0.1 & 55 \\
\hline SR-CRSP-15 $^{\mathbf{2 k}}$ & {$[15]$} & $\begin{array}{l}\text { CTTTACTTCTGACATGGTATTTCCC } \\
\text { TGCCACTCAATTTAGCAAGC }\end{array}$ & Caprine & Unknown & 0.4 & 55 \\
\hline TGLA126 $^{\mathbf{2 1}}$ & {$[9]$} & $\begin{array}{l}\text { CTAATTTAGAATGAGAGAGGCTTCT } \\
\text { TTGGTCTCTATTCTCTGAATATTCC }\end{array}$ & Bovine & 20 & 0.2 & 45 \\
\hline BM4505 $^{\mathbf{2 1}}$ & {$[3]$} & $\begin{array}{l}\text { TTATCTTGGCTTCTGGGTGC } \\
\text { ATCTTCACTTGGGATGCAGG }\end{array}$ & Bovine & 26 & 0.4 & 45 \\
\hline SR-CRSP-23 $^{\mathbf{2 m}}$ & {$[31]$} & $\begin{array}{l}\text { TGAACGGGTAAAGATGTG } \\
\text { TGTTTTTAATGGCTGAGTAG }\end{array}$ & Caprine & Unknown & 0.2 & 45 \\
\hline INRA040 $^{\mathbf{2 m}}$ & {$[12]$} & $\begin{array}{l}\text { TCAGTCTCCAGGAGAGAAAAC } \\
\text { CTCTGCCCTGGGGATGATTG }\end{array}$ & Bovine & 2 & 0.4 & 45 \\
\hline INRABERN175 $^{\mathbf{2 m}}$ & {$[28]$} & $\begin{array}{l}\text { TGATGAGGATGGATGCTAACT } \\
\text { CTGCAAATAAGAAAACTGAATAAA }\end{array}$ & Bovine & 25 & 0.5 & 45 \\
\hline
\end{tabular}


Table II. Unbiased heterozygosity ( $H_{n \text {.b. }}$ Nei 1978$)$, observed heterozygosity $\left(H_{\circ}\right)$, and allele size-range for each species. Exponent letters indicate multiplex PCR, exponent numbers indicate loci multiplex loaded together in one well. "“" indicate data not available.

\begin{tabular}{|c|c|c|c|c|c|c|c|c|c|}
\hline \multirow[b]{2}{*}{ Locus } & \multicolumn{3}{|c|}{ C.i. ibex $(n=36)$} & \multicolumn{3}{|c|}{ C. [i.] sibirica $(n=4)$} & \multicolumn{3}{|c|}{ C. pyrenaica $(n=4)$} \\
\hline & $\mathrm{H}_{\mathrm{n} \cdot \mathrm{b}}$ & $\mathrm{H}_{\mathrm{o}}$ & $\begin{array}{l}\text { Size range } \\
\text { / No. of alleles }\end{array}$ & $\mathrm{H}_{\mathrm{n} \cdot \mathrm{b}}$ & $\mathrm{H}_{\mathrm{o}}$ & $\begin{array}{l}\text { Size range } \\
\text { / No. of alleles }\end{array}$ & $\mathrm{H}_{\mathrm{n} \cdot \mathrm{b}}$ & $\mathrm{H}_{\mathrm{O}}$ & $\begin{array}{l}\text { Size range } \\
/ \text { No. of alleles }\end{array}$ \\
\hline OarFCB0193 $^{1 \mathrm{a}}$ & 0.56 & 0.47 & $135-145 / 4$ & 0.75 & 1.00 & $113-123 / 4$ & 0.82 & 0.75 & $119-127 / 4$ \\
\hline OarFCB48 ${ }^{1 a}$ & 0.43 & 0.48 & $152-158 / 2$ & 0.86 & 0.75 & $154-168 / 5$ & 0.00 & 0.00 & $152 / 1$ \\
\hline SR-CRSP-26 ${ }^{1 b}$ & 0.51 & 0.42 & $133-139 / 2$ & 0.54 & 0.25 & $133-139 / 2$ & 0.25 & 0.25 & $137-139 / 2$ \\
\hline ETH10 $1 \mathrm{~b}$ & 0.49 & 0.45 & $211-215 / 3$ & 0.46 & 0.50 & $207-209 / 3$ & 0.00 & 0.00 & $201 / 1$ \\
\hline ILSTS029 $^{1 \mathrm{c}}$ & 0.26 & 0.24 & $173-175 / 2$ & 0.89 & 0.50 & $169-183 / 5$ & 0.75 & 0.25 & $173-187 / 3$ \\
\hline BM1818 $1 d$ & 0.26 & 0.24 & $262-266 / 2$ & 0.43 & 0.50 & $254-256 / 2$ & 0.43 & 0.50 & $258-260 / 2$ \\
\hline INRABERN185 $1 \mathrm{~d}$ & 0.69 & 0.64 & $268-274 / 4$ & 0.73 & 0.33 & $268-292 / 3$ & 0.46 & 0.25 & $268-274 / 3$ \\
\hline $\mathrm{BM}^{2113^{1 e}}$ & 0.66 & 0.55 & $142-158 / 5$ & 0.53 & 0.67 & $140-142 / 2$ & 0.54 & 0.25 & $152-156 / 2$ \\
\hline SR-CRSP-25 ${ }^{1 f}$ & 0.52 & 0.48 & $104-120 / 3$ & 0.46 & 0.50 & $106-112 / 3$ & 0.54 & 0.75 & $112-114 / 2$ \\
\hline HEL $1^{1 f}$ & 0.52 & 0.55 & $122-126 / 3$ & 0.57 & 1.00 & $118-144 / 2$ & 0.68 & 1.00 & $126-142 / 3$ \\
\hline SR-CRSP-6 ${ }^{1 \mathrm{~g}}$ & 0.03 & 0.03 & $157-159 / 2$ & 0.75 & 0.75 & $151-155 / 3$ & 0.57 & 0.50 & $155-157 / 2$ \\
\hline SR-CRSP-9 ${ }^{1 g}$ & 0.40 & 0.36 & $213-225 / 3$ & 0.43 & 0.50 & $219-221 / 2$ & 0.00 & 0.00 & $213 / 1$ \\
\hline OarAE054 $4^{2 h}$ & 0.54 & 0.42 & $125-129 / 3$ & 0.82 & 0.75 & $125-131 / 4$ & 0.46 & 0.50 & $121-131 / 3$ \\
\hline TGLA122 $2 h$ & 0.48 & 0.45 & $137-155 / 4$ & 0.86 & 1.00 & $137-155$ / 4 & 0.54 & 0.75 & $145-155 / 2$ \\
\hline HAUT27 ${ }^{2 i}$ & 0.59 & 0.64 & $134-140 / 3$ & 0.79 & 0.75 & $132-142 / 4$ & 0.43 & 0.50 & $132-134 / 2$ \\
\hline ILSTSO30 $^{2 j}$ & 0.24 & 0.27 & $165-175 / 3$ & 0.93 & 0.75 & $157-175 / 6$ & 0.43 & 0.00 & $169-173 / 2$ \\
\hline SR-CRSP-8 ${ }^{2 j}$ & 0.51 & 0.55 & $231-233 / 2$ & 0.89 & 0.75 & $221-247 / 5$ & 0.79 & 0.75 & $239-251 / 5$ \\
\hline SR-CRSP-24 ${ }^{2 k}$ & 0.52 & 0.09 & $150-170 / 4$ & 0.68 & 0.50 & $156-164 / 3$ & 0.64 & 0.50 & $156-162 / 4$ \\
\hline SR-CRSP-15 ${ }^{2 k}$ & 0.42 & 0.36 & $184-196 / 3$ & 0.54 & 0.75 & $186-190 / 2$ & 0.79 & 0.75 & $184-192 / 4$ \\
\hline TGLA126 ${ }^{21}$ & 0.66 & 0.61 & $187-201 / 3$ & 0.61 & 0.75 & $287-295 / 3$ & 0.57 & 0.50 & $203-209 / 2$ \\
\hline $\mathrm{BM}^{4505^{21}}$ & 0.44 & 0.42 & $280-286 / 3$ & 0.82 & 0.75 & $258-278 / 4$ & 0.68 & 0.50 & $272-280 / 3$ \\
\hline SR-CRSP-23 ${ }^{2 m}$ & 0.36 & 0.34 & $089-095 / 2$ & 0.96 & 1.00 & $085-099 / 7$ & 0.89 & 0.75 & $081-095 / 5$ \\
\hline INRA040 $^{2 m}$ & 0.66 & 0.34 & $227-257 / 4$ & 0.25 & 0.25 & $229-237 / 2$ & 0.54 & 0.25 & $251-261 / 2$ \\
\hline INRABERN175 $2 \mathrm{~m}$ & - & - & - & 0.89 & 0.75 & $138-170 / 5$ & 0.71 & 0.50 & $152-158 / 3$ \\
\hline $\begin{array}{l}\text { Av. heterozygosity } \\
\text { (Hs) }\end{array}$ & 0.44 & 0.40 & & 0.69 & 0.67 & & 0.52 & 0.45 & \\
\hline
\end{tabular}




\section{REFERENCES}

[1] Allendorf F., Ryman N., The role of genetics in population variability analysis, in: Population Viability Analysis, Beissinger S. R. and McCullough D.R. (in press).

[2] Bhebhe E., Kogi J., Holder D.A., Arevalo E., Derr J.N., Linn R.A., Davis S.K., Taylor J.F., Ruvuna F., Caprine microsatellite dinucleotide repeat polymorphisms at the SR-CRSP-6, SR-CRSP-7, SR-CRSP-8, SR-CRSP-9, and SRCRSP-10 loci, Anim. Genet. 25 (1994) 203.

[3] Bishop M.D., Kappes S.M., Keele J.W., Stone R.T., Sunden S.L.F., Hawkins G.A., Solinas-Toldo S., Fries R., Grosz M.D., Yoo J., Beattie C.W., A genetic linkage map for cattle, Genetics 136 (1994) 619-639.

[4] Bruford M.W., Wayne R.K., Microsatellites and their application to population genetic studies, Gen. Evol. (1993) 939-942.

[5] Buchanan F.C., Crawford A.M., Ovine microsatellites at the OarFCB11, OarFCB128, OarFCB193, OarFCB266, and OarFCB304 loci, Anim. Genet. 24 (1993) 145.

[6] Buchanan F.C., Galloway S.M., Crawford A.M., Ovine microsatellites at the OarFCB5, OarFCB19, OarFCB20, OarFCB48, OarFCB129 and OarFCB226 loci, Anim. Genet. 25 (1991) 60.

[7] Forbes S.H., Hogg J.T., Buchanan F.C., Crawford A.M., Allendorf F.W., Microsatellite evolution in congeneric mammals: Domestic and bighorn sheep, Mol. Biol. Evol. (1995) 1106-1113.

[8] Garcia-Moreno J., Matocq M.C., Roy M.S., Geffen E., Wayne R.K., Relationships and genetic purity of the endangered Mexican wolf based on analysis of microsatellite loci, Conserv. Biol. 10 (1996) 376-389.

[9] Georges M., Massey J.M., Polymorphic DNA markers in Bovidae, Patent WO 92/13102, 1992.

[10] Hassanin A., Pasquet A., Vigne J.D., Molecular systematics of the subfamily Caprinae (Artiodactyla, Bovidae) as determined from cytochrome b sequences, J. Mamm. Evol. 5 (1998) 217-236.

[11] Houlden B.A., England P.R., Taylor A.C., Greville W.D., Low genetic variability of Koala Phascolarctos cinereus in south-eastern Australia following a severe population bottleneck, Mol. Ecol. 5 (1996) 269-281.

[12] Kappes S.M., Keele J.W., Stone R.T., McGraw R.A., Sonstegard T.S., Smith T.P., Lopez-Corrales N.L., Beattie C.W., A second-generation linkage map of the bovine genome, Genome Res. 7 (1997) 235-249.

[13] Kaukinen J., Varvio S.L., Eight polymorphic bovine microsatellites, Anim. Genet. 24 (1993) 148.

[14] Kemp S.J., Hishida O., Wambugu J., Rink A., Longeri M., Ma R.Z., Da Y., Lewin H.A., Barendse W., Teale A.J., A panel of polymorphic bovine, ovine and caprine microsatellite markers, Anim. Genet. 26 (1995) 299-306.

[15] Kogi J., Yeh C.C., Bhebhe E., Burns B.M., Ruvuna F., Davis S.K., Taylor J.F., Caprine microsatellite dinucleotide repeat polymorphisms at the SR-CRSP-11, SR-CRSP-12, SR-CRSP-13, SR-CRSP-14 and SR-CRSP-15 loci., Anim. Genet. 26 (1995) 449. 
[16] Luikart G., Biju-Duval M.-P., Ertugrul O., Zagdsuren Y., Maudet C., Taberlet P., Power of 22 microsatellite makers in fluorescent multiplexes for parentage testing in goats (Capra hircus), Anim. Genet. (1999) 431-438.

[17] Moore S.S., Sargeant L.L., King T.J., Mattick J.S., Georges M., Hetzel D.J., The conservation of dinucleotide microsatellites among mammalian genomes allows the use of heterologous PCR primer pairs in closely related species, Genomics 10 (1991) 654-660.

[18] Paetkau D., Strobeck C., Microsatellite analysis of genetic variation in black bear populations, Mol. Ecol. 3 (1994) 489-495.

[19] Penty J.M., Henry H.M., Ede A.J., Crawford A.M., Ovine microsatellites at the OarAE16, OarAE54, OarAE57, OarAE119 and OarAE129 loci, Anim. Genet. 24 (1993) 219.

[20] Roed K.H., Microsatellite variation in Scandinavian Cervidae using primers derived from Bovidae, Hereditas 129 (1998) 19-25.

[21] Saitbekova N., Gaillard C., Obexer-Ruff G., Dolf G., Genetic diversity in Swiss goat breeds based on microsatellite analysis, Anim. Genet. 30 (1999) 36-41.

[22] Shackleton D.M., The IUCN/SSC Caprinae Specialist Group in Wild sheep and goat and their relatives: Status survey and conservation action plan for Caprinae, IUCN, Switzerland and Cambridge, 1997.

[23] Solinas Toldo S., Fries R., Steffen P., Neibergs H.L., Barendse W., Womack J.E., Hetzel J.D., Stranzinger G., Physically mapped cosmid-derived microsatellite markers as anchor loci on bovine chromosomes, Mamm. Genome (1993) 720727.

[24] Stüwe M., Scribner K.T., Low genetic variability in reintroduced alpine ibex (Capra ibex ibex) populations, J. Mamm. 70 (1989) 370-373.

[25] Taberlet P., Luikart G., Non-invasive genetic sampling and individual identification, Biol. J. Linnean Soc. 68 (1999).

[26] Taylor A.C., Sherwin W.B., Wayne R.K., Genetic variation of microsatellite loci in a bottlenecked species: The hairy-nosed wombat Lasiorhinus krefftii, Mol. Ecol. 3 (1994) 277-290.

[27] Thieven U., Solinas-Toldo S., Friedl R., Masabanda J., Fries R., Barendse W., Simon D., Harlizius B., Polymorphic CA-microsatellites for the integration of the bovine genetic and physical map, Mamm. Genome 8 (1997) 52-55.

[28] Vaiman D., Schibler L., Bourgeois F., Oustry A., Amigues Y., Cribiu E.P., A genetic linkage map of the male goat genome, Genetics 144 (1996) 279-305.

[29] Wilson G.A., Stronbeck C., Wu L., Coffin J.W., Characterization of microsatellites loci in caribou Rangifer tarandus, and their use in other artiodactyls, Mol. Ecol. 6 (1997) 697-699.

[30] Yang L., Zhao S., Li K., Peng Z.Z., Montgomery G.W., Determination of genetic relationships among five indigenous Chinese goat breeds with six microsatellite markers, Anim. Genet. 6 (1999) 452-455.

[31] Yeb C., Kogi J.K., Holder M., Guerra T.M., Davis S.K., Taylor J.F., Caprine microsatellite dinucleotide repeat polymorphisms at the SR-CRSP 21, 22, 23, 24, 25, 26, and 27 loci, Anim. Genet. 28 (1997) 370-371. 